\title{
Radiosurgery and stereotactic radiotherapy for intracranial meningiomas
}

\author{
Rodrigo Couto Torres, M.D., Leonardo Frighetto, M.D., \\ Antonio A. F. De Salles, M.D., Ph.D., Brian Goss, M.D., Paul Medin, Ph.D., \\ Timothy Solberg, Ph.D., Judith Marianne Ford, M.D., Ph.D., \\ AND Michael SELCH, M.D.
}

Division of Neurosurgery and Department of Radiation Oncology, University of California at Los Angeles, California

\begin{abstract}
Object. The authors report the evolution of linear accelerator (LINAC)-based radiosurgery in the treatment of patients with intracranial meningiomas. They describe the technical aspects as well as clinical and radiological outcomes.

Methods. The authors performed a retrospective review of 161 patients harboring 194 intracranial meningiomas treated with various types of stereotactic irradiation at their institution between May 1991 and July 2002. Clinical and radiological follow-up data (mean follow-up period 32.5 months, range 6-125 months) were obtained in 128 patients $(79.5 \%)$ with 156 meningiomas $(80.4 \%)$. There were 88 women and 40 men whose mean age was 57.2 years (range 18-87 years). Stereotactic irradiation was the primary treatment in 44 patients, and 84 patients underwent resection prior to radiosurgery. Stereotactic radiosurgery (SRS) was used to treat 79 lesions and fractionated stereotactic radiotherapy (SRT) was used to treat 77. The mean dose for SRS was 1567 cGy (range 1200-2285 cGy) prescribed to a mean isodose line of $66.6 \%$ (range 50-90\%). Stereotactic radiotherapy was delivered using a mean dose of 4839 cGy (range 2380-5400 cGy), prescribed to a mean isodose line of 89\% (range 50-90\%).

The mean follow-up periods were 40 and 24 months in SRS- and SRT-treated patients, respectively. Tumor control was achieved in 58 SRT-treated benign meningiomas (90\%) and in 70 SRT-treated lesions (97.2\%). In patients with atypical meningiomas a considerably poorer prognosis was seen. Clinical improvement or stabilization of symptoms was observed in the majority of patients. Symptomatic complications were limited to four patients $(5 \%)$ treated with SRS and four $(5.2 \%)$ treated with SRT.

Conclusions. Stereotactic irradiation techniques have changed the neurosurgical approach to intracranial meningiomas. Either SRS or SRT delivered as a primary treatment in selected cases of skull base lesions or as an adjuvant after conservative resection has improved the management of these complex intracranial tumors.
\end{abstract}

\section{KEY WORDS • linear accelerator • stereotactic radiosurgery • meningioma • stereotactic radiotherapy}

A complete resection including dural attachment and areas of bone invasion remains the best treatment for intracranial meningiomas. Although in many anatomical locations a gross-total resection can be achieved with little morbidity, the same good results cannot be reproduced in the treatment of lesions involving eloquent anatomical structures. Morbidity rates of 12 to $30 \%$ have been reported despite the recent advances in microsurgical techniques. ${ }^{3,4,6,8,20,27}$ To preserve neurological function, incomplete excision is frequently performed. After complete resection, expected 5- and 10-year recurrence rates are 7 and $20 \%$, respectively. ${ }^{13,19}$

Abbreviations used in this paper: $\mathrm{CT}=$ computerized tomography; EBRT $=$ external-beam radiotherapy; LINAC $=$ linear accelerator; $\mathrm{MR}=$ magnetic resonance; $\mathrm{PFS}$ = progression-free survival; SRS = stereotactic radiosurgery; SRT = stereotactic radiotherapy; $\mathrm{UCLA}=$ University of California at Los Angeles.
Initially, irradiation was applied in the management of residual tumors after microsurgery. Its appropriate application proved to be successful when using all the available techniques, including LINAC, ${ }^{7,12,28,29}$ gamma knife, ${ }^{13,15,16,30}$ proton-beam, ${ }^{34}$ and conventional fractionated EBRT. 2,10,11,23,32

The high success rate and safety demonstrated with SRS after the advent of high-definition MR imaging established this modality as an invaluable tool in the management of intracranial meningiomas. The widespread use of stereotactic irradiation techniques has changed the neurosurgical approach to intracranial meningiomas, especially in relation to complex skull base lesions. Recent advances in stereotactic irradiation delivery continue to enhance the applications of these techniques to lesions previously not suitable for SRS. These advances include the advent of fractionated SRT and the availability of a micromultileaf collimator capable of shaped-beam SRS and SRT. 
We describe our experience at UCLA with the management of intracranial meningiomas, demonstrating the evolution of the treatment planning and radiation delivery in the last decade.

\section{CLINICAL MATERIAL AND METHODS}

Between 1991 and 2002, 161 patients with 194 intracranial meningiomas underwent SRS or fractionated SRT at UCLA Medical Center. All procedures were performed using a LINAC-based stereotactic system. Initially, treatments were performed using a Philips SRS 200 system adapted into a Clinac 18 LINAC (Varian, Palo Alto, CA). A dedicated LINAC for radiosurgery was first used in 1996- the X-Knife system (Radionics, Burlington, MA). Since 1997 our department has used the Novalis (Brainlab, Heimstetten, Germany) dedicated LINAC. Clinical and radiological follow-up data were obtained in 128 patients $(79.5 \%)$ harboring 156 meningiomas $(80.4 \%)$. Stereotactic radiosurgery was the modality of choice in 79 meningiomas $(50.6 \%)$, whereas $77(49.4 \%)$ were treated with fractionated SRT. The overall mean follow-up period was 32.5 months (range 6-125 months). The mean follow-up period for SRS-treated patients was 40.6 months (range 6-125 months) and that for SRT-treated patients was 23.8 months (range 6-72 months). There were 88 women $(68.7 \%)$ and 40 men $(31.2 \%)$ whose mean age was 57.2 years (range $18-87$ years). Stereotactic irradiation was the first treatment option in 44 patients $(34.4 \%)$ and was delivered following microsurgery in $84(65.6 \%)$.

Routinely, a BRW (Radionics) or a BrainLab (BrainLab) stereotactic frame was applied after administration of a local anesthetic. A stereotactic CT scan was obtained and fused with a previously acquired 3-mm slice MR image. Image fusion was performed using a mutual information algorithm, which allows geometrical alignment between the two image sets. The CT scans were used for the precision of their stereotactic localization, whereas highresolution MR images provided the anatomical details necessary for treatment planning. The same protocol was used for SRT-treated patients; however, a replaceable mask made of thermotransformable material (BrainLab) or a GTC frame (Radionics) was applied.

Complex-shaped lesions were treated with conformal planning that involved the use of multiple isocenters, arc selection, and weighting to avoid critical structures. Recently a micromultileaf collimator-generated conformal plan has been used, obviating the need for multiple isocenters for dose conformation. The dosimetry characteristics used in this series are presented in Table 1. Stereotactic radiotherapy was indicated for tumors involving the optic apparatus, substantially compressing the brainstem, or those deemed too large for SRS treatment. Its selection was also based on the UCLA classification of sellar and parasellar meningiomas (Table 2). Follow-up evaluation was performed with MR imaging every 6 months for the first 3 years and every 12 months thereafter.

\section{RESULTS}

The overall tumor growth control rate for the 135 benign meningiomas in 128 patients was $94.8 \%$. Reduction
TABLE 1

Technical parameters used in the treatment of 156 intracranial meningiomas*

\begin{tabular}{lcc}
\hline \hline \multirow{2}{*}{\multicolumn{1}{c}{ Parameter }} & \multicolumn{2}{c}{ Value (range) } \\
\cline { 2 - 3 } & SRS (79 lesions) & SRT (77 lesions) \\
\hline mean no. of fractions & 1 & $26.85(5-30)$ \\
mean volume (ml) & $12.7(1.1-43)$ & $16.1(1.25-57)$ \\
mean isodose (\%) & $66.6(50-90)$ & $89.27(50-100)$ \\
mean prescribed dose & $1567(1200-2285)$ & $4839(2380-5400)$ \\
mean max dose & $2456(1500-4000)$ & $5350(4500-6000)$ \\
mean dose/fraction (cGy) & NA & $183(165-500)$ \\
multiple isocenters & 53 & 7 \\
shaped beam & 26 & 70 \\
CT planning only & 15 & 0 \\
\hline
\end{tabular}

* NA $=$ not applicable.

in tumor volume was observed in 46 lesions (34.1\%), stabilization of the tumor with no evidence of growth in 82 lesions $(60.7 \%)$, and further tumor growth in seven lesions $(5.2 \%)$.

Single-dose SRS provided a treatment success rate of $92 \%$ after a mean follow-up period of 40.6 months. Failure was observed in five $(7.9 \%)$ of the 63 patients treated with this modality. Tumor volume reduction occurred in 22 patients $(34.9 \%)$ and stabilization in 36 patients $(57.1 \%)$ in the SRS group. Fractionated SRT was associated with a treatment success rate of $97.2 \%$ after a mean follow-up period of 23.8 months. Further tumor growth was observed in two (2.8\%) of the 72 patients in this group. Reduction in tumor volume occurred in $24(33.8 \%)$ and stabilization in $46(63.4 \%)$ of the patients treated with SRT (Fig. 1). The tumor growth control for benign intracranial meningiomas is summarized in Table 3.

Four patients with benign meningiomas underwent open surgery after SRS because the tumor growth was not controlled. Eight patients with benign lesions that recurred underwent a new SRS session. Only one patient harboring an atypical meningioma previously treated with SRT then underwent open resection after tumor growth was observed.

The same tumor control rate could not be achieved when treating meningiomas that presented with atypical features (Table 4). In the present series, the overall tumor growth control for atypical meningiomas was $38.1 \%$ (eight patients). Reduction in tumor size was observed in two cases (9.5\%) - one SRS- and one SRT-treated patient. Tumor stabilization occurred in four $(25 \%)$ of the SRS- and two $(40 \%)$ of the SRT-treated patients. Tumor progression was observed in $62 \%$ (13 patients)-11 (68.7\%) treated with SRS and two (40\%) treated with SRT (Table 3).

Follow-up neurological and imaging data were available in 128 patients in a period ranging from 6 months to 10 years (mean 32.5 months, median 24 months) (Table 5). Improvement of the presenting symptoms was observed in 22 SRS-treated patients (35\%) and 21 SRT-treated patients (32\%) (Fig. 2). The overall rate of clinical improvement was $33.5 \%$ (43 patients). The clinical status remained unchanged in $36(57.1 \%)$ and 42 patients (64.6\%) who underwent SRS and SRT, respectively. Worsening of the clinical symptoms was observed in five pa- 
TABLE 2

The UCLA radiosurgery grading system for cavernous sinus/parasellar meningiomas

\begin{tabular}{cc}
\hline \hline Grade & Meningioma Radiological Aspect \\
\hline I & $\begin{array}{c}\text { confined to the cavernous sinus/parasellar region } \\
\text { involvement of the petroclival region w/o brainstem com- } \\
\text { pression } \\
\text { extension to \& compression of the optic nerve, chiasm, or } \\
\text { tract }\end{array}$ \\
IV & $\begin{array}{c}\text { involvement of the petroclival region w/ compression of the } \\
\text { brainstem }\end{array}$ \\
V & extensive involvement of both cavernous sinuses
\end{tabular}

tients $(7.9 \%)$ who underwent SRS and in two patients $(3.1 \%)$ who underwent SRT due to complications or progression of the disease.

The incidence of clinical complications in the SRS group was $5 \%$ (four procedures). Slight decrease in visual acuity was observed in two and decrease in facial sensation in another two cases. Imaging-detected abnormalities not proceeded by clinical symptoms were detected after three procedures $(3.7 \%)$. Radiation-induced changes in the pattern of contrast enhancement due to disruption of the blood-brain barrier were observed on two images and a small area of radiation necrosis on one follow-up image.

In the SRT group, symptomatic complications occurred after four procedures (5.2\%). Mild reduction in facial sensation was observed in three and subjective complaint of worsened diplopia in one case. Considering all procedures included in this study, the overall incidence of symptomatic complications was $5.1 \%$. None of the patients needed further surgical treatment related to the occurrence of complications. These complications were mild and did not alter patients' activities of daily living.

\section{DISCUSSION}

In the present study we found that both SRS and frac-

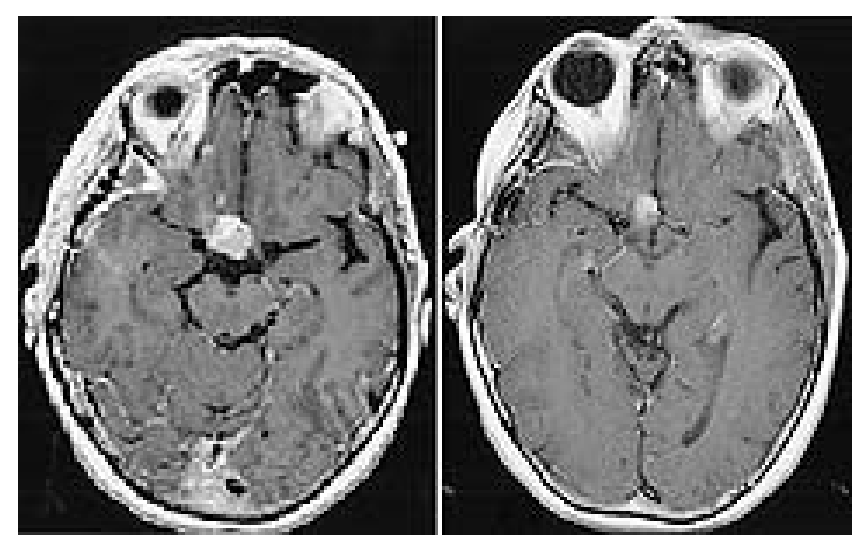

Fig. 1. Left: Preoperative MR image. Right: Control 24month postoperative MR image demonstrating a significant decrease in the size of an intracranial meningioma after SRT. A single shaped-beam isocenter, 28 fractions of $180 \mathrm{cGy}$, prescribed to the $90 \%$ isodose line were applied for a total dose of 5040 cGy. Improved visual function was demonstrated during follow up. (Fig. 2)
TABLE 3

Tumor growth control after stereotactic radiation of benign meningiomas

\begin{tabular}{lccc}
\hline \hline & \multicolumn{2}{c}{ Treatment Group (\%) } & \\
\cline { 2 - 3 } \multicolumn{1}{c}{ Tumor Size } & SRS & SRT & Total (\%) \\
\hline decreased & $22(34.9)$ & $24(33.3)$ & $46(34.1)$ \\
no change & $36(57.1)$ & $46(63.8)$ & $82(60.7)$ \\
increased & $5(7.9)$ & $2(2.7)$ & $7(5.2)$ \\
total & $63(100)$ & $72(100)$ & $135(100)$ \\
tumor control & $58(92)$ & $70(97.2)$ & $128(94.8)$ \\
\hline
\end{tabular}

tionated SRT are effective as an adjuvant or as a primary treatment for intracranial meningiomas. As demonstrated in the literature, success rates of greater than $90 \%$ and with few associated complications can be achieved when stereotactic irradiation is performed in the management of intracranial meningiomas. ${ }^{7-9,12,13,15,16,30}$ A true comparison between SRS and SRT should not be made and is not the goal of this report. Both modalities are used in different clinical scenarios in our department and should be available to complement each other, providing safer treatment options for patients (Fig. 3). The slight difference in tumor control in this study for patients treated with SRS or SRT does not reflect advantages of a specific modality. It is likely a factor related to the longer follow-up interval available for the SRS group, as well as the improvement of imaging and planning over the years. For instance, the first $15 \mathrm{SRS}$-treated patients underwent treatment planning based on CT scans only. We also found that SRS can be indicated for larger tumors not previously considered candidates. Tumors encasing or compressing important structures such as the optic apparatus, cranial nerves and brainstem can also be treated, benefiting from the radiobiological advantages of SRT.

Initially, irradiation was an adjuvant used for residual tumor after microsurgery. ${ }^{2}$ The low morbidity and mortality rates achieved using SRS and SRT compared with those associated with attempts at complete resection ${ }^{14,27}$ and conventional radiotherapy have led to an increase of the use of stereotactic irradiation for intracranial meningiomas. ${ }^{4,5,9,12,13,16,19,20,26,28,33,35}$ In the management of smallto-moderate sized meningiomas $(<35 \mathrm{~mm})$ without symptomatic mass effect, radiosurgery has achieved a similar actuarial PFS interval as that seen for completely resected meningiomas, including their dural attachments and bone abnormalities (Simpson Grade 1). Radiosurgery

TABLE 4

Tumor growth control after stereotactic radiation of atypical meningiomas

\begin{tabular}{lccc}
\hline \hline & \multicolumn{2}{c}{ Treatment Group (\%) } & \\
\cline { 2 - 3 } \multicolumn{1}{c}{ Tumor Size } & SRS & SRT & Total (\%) \\
\hline decreased & $1(6.2)$ & $1(20)$ & $2(9.5)$ \\
no change & $4(25)$ & $2(40)$ & $6(28.5)$ \\
increased & $11(68.7)$ & $2(40)$ & $13(62)$ \\
total & $16(100)$ & $5(100)$ & $21(100)$ \\
tumor control & $5(31.2)$ & $3(60)$ & $8(38.1)$ \\
\hline
\end{tabular}


TABLE 5

Clinical follow-up results

\begin{tabular}{lccc}
\hline \hline \multirow{2}{*}{$\begin{array}{c}\text { Neurological } \\
\text { Findings }\end{array}$} & \multicolumn{2}{c}{ Treatment Group (\%) } & \\
\cline { 2 - 3 } & \multicolumn{1}{c}{ SRS } & SRT & Total (\%) \\
\hline improved & $22(35)$ & $21(32.3)$ & $43(33.5)$ \\
unchanged & $36(57.1)$ & $42(64.6)$ & $78(60.9)$ \\
worsened & $5(7.9)$ & $2(3.1)$ & $7(5.4)$ \\
no. of cases & $63(100)$ & $65(100)$ & $128(100)$ \\
\hline
\end{tabular}

has provided a higher PFS interval compared with that in patients in whom resections were Simpson Grades 2, 3 , and $4 .^{25}$

Microsurgery remains the best option for symptomatic intracranial meningiomas when complete resection can be achieved with low morbidity. Depending on the tumor location and complexity of nearby structures, complete excision of intracranial meningiomas, including their dural site of origin, is unlikely without causing morbidity. ${ }^{17,18}$ In such cases, SRS and SRT are options either as an adjuvant or as a first-line treatment. This includes meningiomas involving the dural venous sinus, brainstem, and cranial nerves.

The use of SRS alone has limitations that preclude its application in every case. These limitations are related to tumor size and proximity to eloquent structures especially the optic apparatus. Single-dose SRS is indicated for meningiomas smaller than $3 \mathrm{~cm}$ or $20 \mathrm{ml}$ in volume and with a minimal distance from the optic apparatus of between 2 and $4 \mathrm{~cm} .{ }^{5,9,19,22,31}$ Many alternatives were used to overcome such limitations, usually resulting in treatment failure. This included two-staged procedures ${ }^{13,20,24}$ or a decrease in the ideal peripheral dose. ${ }^{20}$

With the advent of SRT such limitations were overcome, partly because it was possible to use this modality alone in the management of large intracranial meningiomas. Stereotactic radiotherapy is ideal for tumors that cannot be completely resected or those for which the resection is associated with a great risk of morbidity and mortality. Because stereotactic irradiation allows the delivery of radiation to larger areas, including the entire postoperative tumor bed, it can also improve the control rates in cases involving subtotally resected meningiomas. Stereotactic radiotherapy is a treatment option that may be undertaken when the risk of SRS is unacceptably high for instance, in cases in which tumors involve the optic pathways and the brainstem. ${ }^{1,33}$ This is especially important in light of the observation of improved visual acuity after SRT. ${ }^{1}$ The deliberate delivery of toxic radiation doses to the optic structures to induce tumor control at the cost of visual loss ${ }^{26,35}$ is no longer acceptable because of the availability of safer modalities. The safety of SRT has already been demonstrated in the treatment of optic nerve sheath meningiomas. Andrews, et al., ${ }^{1}$ reported on 33 patients treated with a paradigm similar to the one used in our institution. In their series preserved vision was demonstrated in $92 \%$ and improved visual acuity in $42 \%$ of cases.

In the case of sellar and parasellar meningiomas, a grading system has been developed to define better the most
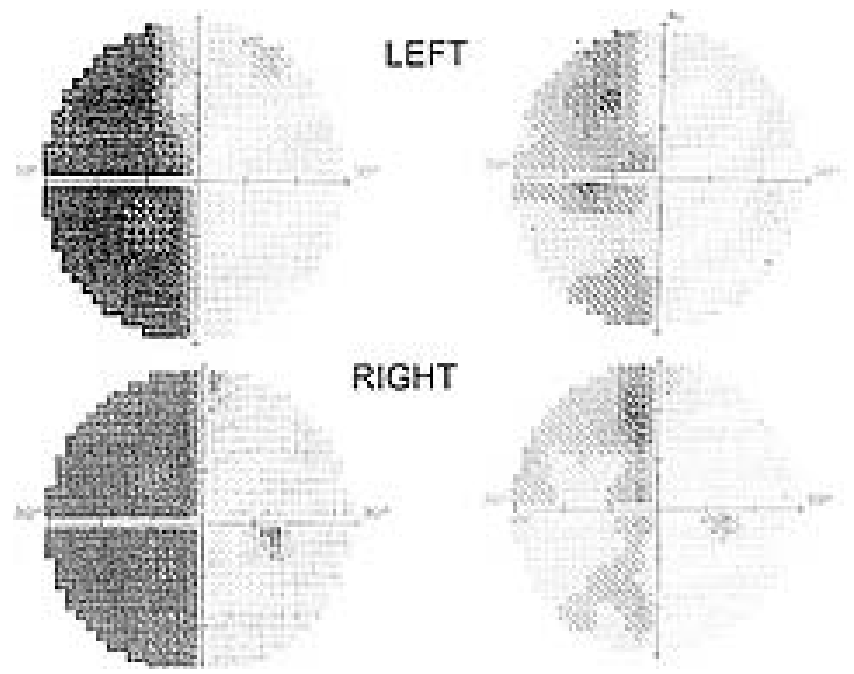

Fig. 2. Initial and 48-month follow-up visual fields obtained in the same patient reported in Fig. 1. Improvement in visual function can be observed.

appropriate case for SRS or SRT. ${ }^{7}$ According to this classification (Table 2), we recommend SRS for low-grade tumors (Grades 1 and 2) and SRT for high-grade tumors (Grades 3-5).

Although there are no long-term follow-up data for patients who underwent SRT for intracranial meningiomas, the expected success rate should be similar to that achieved using conventional EBRT, with fewer complications. A 5- and 10-year PFS rate of 92 and 83\%, respectively, has been reported after EBRT. ${ }^{23}$ Although effective and associated with a low morbidity rate, complications including radiation retinopathy, cataract, hypopituitarism, and short-term memory deficit occurred in $13.4 \%$ of the patients. ${ }^{23}$

Analysis of the data presented in this report as well as that in the literature supports the assertion that stereotactic irradiation alone can provide similar results as microsurgery followed by radiotherapy. ${ }^{2,7,9,10,13,15,16,30,32}$ Thus, the use of SRS or SRT as the first-line treatment for the management of intracranial meningiomas is feasible. The selection of the best treatment option for these lesions, however, should include consideration of tumor location, severity of presenting symptoms, and the long-term follow-up data of the available modalities. Microsurgery is superior in providing symptomatic relief, and it is the best strategy for meningiomas located in the convexity of the skull and in other locations where a total resection can be achieved. Stereotactic irradiation has proven to be a better modality in cases of complex skull base lesions, especially those involving the cavernous sinus, providing lower recurrence rates and fewer complications.

Moreover, recent advances in stereotactic irradiation techniques can further improve treatment-related outcomes and increase the number of tumors suitable for such treatment. These advances include the advent of SRT and the availability of shaped-beam radiosurgery capable of delivering intensity-modulated SRS/SRT. These treatment modalities produce less target dose heterogeneity because of the use of a single isocenter and shorter estimated treat- 


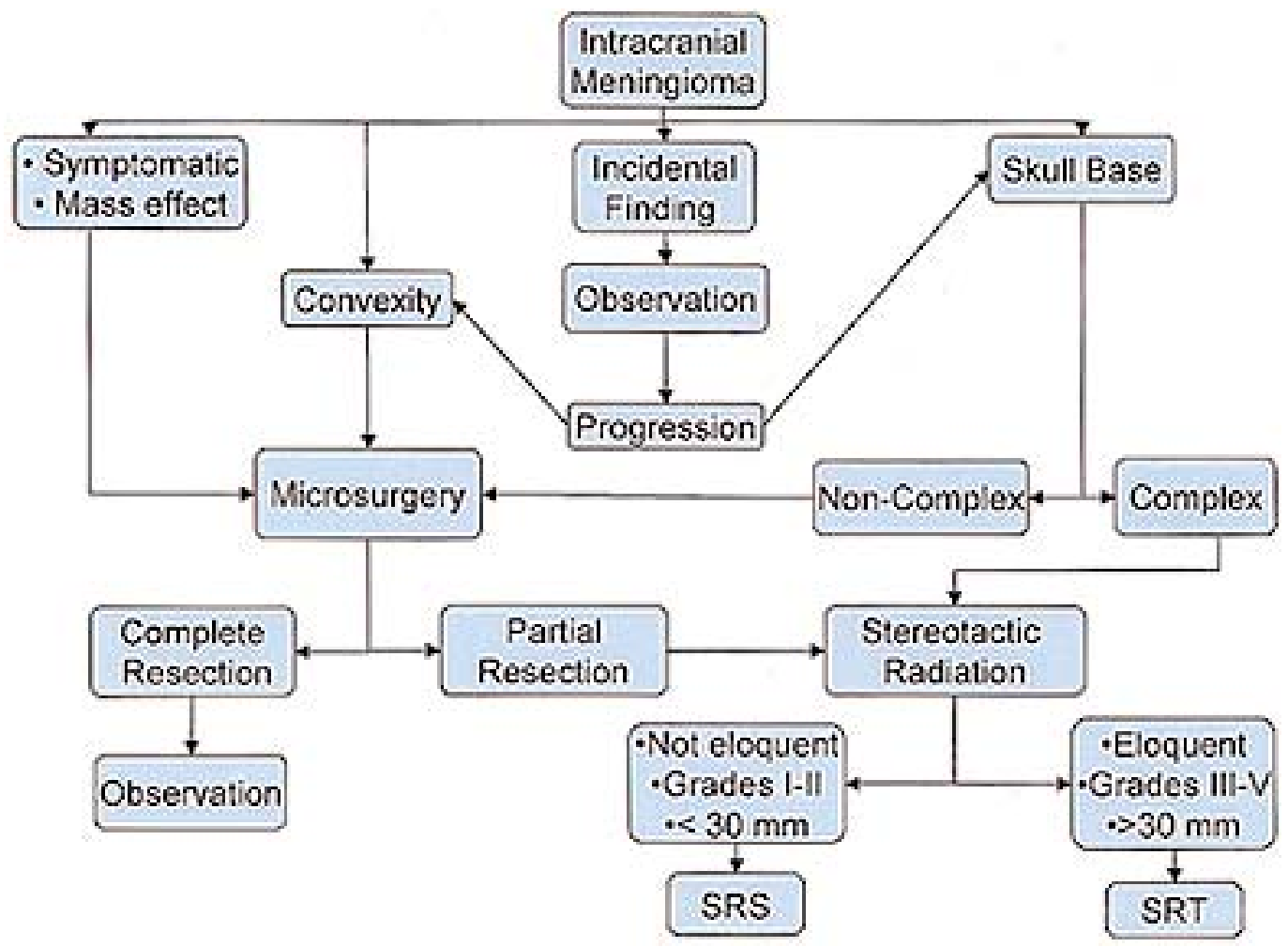

Fig. 3. Flowchart depicting selection criteria for stereotactic irradiation (either SRS or SRT) of intracranial meningiomas used in our clinical practice.

ment times. The application of intensity-modulated SRS or SRT in the treatment of complex skull base lesions has been shown to provide comparable target coverage and sparing of structures at risk, with an improved conformity index at the prescription isodose contour compared with the multiple isocenter techniques. ${ }^{21}$ This modality is important in the treatment of these lesions because the skull base location is at high risk for functional morbidity; additionally the potential benefits of improved conformality may be especially relevant to skull base lesions.

\section{CONCLUSIONS}

Stereotactic irradiation has already been demonstrated to be effective, either as an adjuvant or as the first treatment option in selected tumors. The use of modern irradiation techniques is changing the management of complex intracranial meningiomas. It may be that less radical surgery of cranial nerve-and vascular structure-involved tumors followed by stereotactic irradiation can reduce the incidence of morbidities related to the management of difficult tumors. Further improvements, including intensitymodulated SRS and SRT could promote a greater sparing of normal brain structures.

\section{References}

1. Andrews DW, Faroozan R, Yang BP, et al: Fractionated stereotactic radiotherapy for the treatment of optic nerve sheath meningiomas: preliminary observations of 33 optic nerves in 30 patients with historical comparison to observation with or without prior surgery. Neurosurgery 51:890-904, 2002
2. Barbaro NM, Gutin PH, Wilson CB, et al: Radiation therapy in the treatment of partially resected meningiomas. Neurosurgery 20:525-528, 1987

3. Bitzer M, Topka H, Morgalla M, et al: Tumor-related venous obstruction and development of peritumoral brain edema in meningiomas. Neurosurgery 42:730-737, 1998

4. Chang SD, Adler JR Jr: Treatment of cranial base meningiomas with linear accelerator radiosurgery. Neurosurgery 41: 1019-1027, 1997

5. Chen JC, Giannotta SL, Yu C, et al: Radiosurgical management of benign cavernous sinus tumors: dose profiles and acute complications. Neurosurgery 48:1022-1032, 2001

6. Connell PP, Macdonald RL, Mansur DB, et al: Tumor size predicts control of benign meningiomas treated with radiotherapy. Neurosurgery 44:1194-1200, 1999

7. De Salles AA, Frighetto L, Grande CV, et al: Radiosurgery and stereotactic radiation therapy of skull base meningiomas: proposal of a grading system. Stereotact Funct Neurosurg 76: 218-229, 2001

8. De Salles AAF, Scaduto A, Goetsch SJ: Radiosurgery of benign tumors, in De Salles AAF, Goetsch SJ (eds): Stereotactic Surgery and Radiosurgery. Madison, WI: Medical Physics Publishing, 1993, pp 369-387

9. Duma CM, Lunsford LD, Kondziolka D, et al: Stereotactic radiosurgery of cavernous sinus meningiomas as an addition or alternative to microsurgery. Neurosurgery 32:699-705, 1993

10. Glaholm J, Bloom HJG, Crow JH: The role of radiotherapy in the management of intracranial meningiomas: The Royal Mardsen Hospital experience with 186 patients. Int J Radiat Oncol Biol Phys 18:755-761, 1990

11. Goldsimth BJ, Wara WM. Wilson CB, et al: Postoperative irradiation for subtotally resected meningiomas. A retrospective analysis of 140 patients treated from 1967 to 1990. J Neurosurg 80:195-201, 1994

12. Hakim R, Alexander E III, Loeffler JS, et al: Results of linear 
accelerator-based radiosurgery for intracranial meningiomas. Neurosurgery 42:446-454, 1998

13. Iwai Y, Yamanaka K, Ishiguro T: Gamma knife radiosurgery for the treatment of cavernous sinus meningiomas. Neurosurgery 52:517-524, 2003

14. Knosp E, Perneczky A, Koos WT, et al: Meningiomas of the space of cavernous sinus. Neurosurgery 38:434-444, 1996

15. Kobayashi T, Kida Y, Mori Y: Long-term results of stereotactic gamma radiosurgery of meningiomas. Surg Neurol 55: 325-331, 2001

16. Kondziolka D, Levy E, Niranjan A, et al: Long-term outcomes after meningioma radiosurgery: physician and patient perspectives. J Neurosurg 91:44-50, 1999

17. Kotapka MJ, Kalia KK, Martinez AJ, et al: Infiltration of the carotid artery by cavernous sinus meningioma. J Neurosurg 81:252-255, 1994

18. Larson JJ, van Loveren HR, Balko MG, et al: Evidence of meningioma infiltration into cranial nerves: clinical implications for cavernous sinus meningiomas. J Neurosurg 83: 596-599, 1995

19. Lee JY, Niranjan A, McInerney J, et al: Stereotactic radiosurgery providing long-term tumor control of cavernous sinus meningiomas. J Neurosurg 97:65-72, 2002

20. Liscak R, Simonova G, Vymazal J, et al: Gamma knife radiosurgery of meningiomas in the cavernous sinus region. Acta Neurochir 141:473-480, 1999

21. Nakamura JL, Pirzkall A, Carol MP, et al: Comparison of intensity-modulated radiosurgery with gamma knife radiosurgery for challenging skull base lesions. Int J Radiat Oncol Biol Phys 55:99-109, 2003

22. Nicolato A, Foroni R, Alessandrini F, et al: The role of Gamma Knife radiosurgery in the management of cavernous sinus meningiomas. Int J Radiat Oncol Biol Phys 53:992-1000, 2002

23. Nutting C, Brada M, Brazil L, Sibtain A. et al: Radiotherapy in the treatment of benign meningioma of the skull base. $\mathbf{J}$ Neurosurg 90:823-827, 1999

24. Pendl G, Schrottner O, Eustacchio S, et al: Stereotactic radiosurgery of skull base meningiomas. Minim Invasive Neurosurg 40:87-90, 1997

25. Pollock BE, Stafford SL, Utter A, et al: Stereotactic radio- surgery provides equivalent tumor control to Simpson Grade 1 resection for patients with small- to medium-size meningiomas. Int J Radiat Oncol Biol Phys 55:1000-1005, 2003

26. Roche PH, Regis J, Dufour H, et al: Gamma knife radiosurgery in the management of cavernous sinus meningiomas. J Neurosurg 93:68-73, 2000

27. Sekhar LN, Patel S, Cusimano M, et al: Surgical treatment of meningiomas involving the cavernous sinus: evolving ideas based on a ten year experience. Acta Neurochir Suppl 65: 58-62, 1996

28. Shafron DH, Friedman WA, Buatti JM, et al: Linac radiosurgery for benign meningiomas. Int J Radiat Oncol Biol Phys 43:321-327, 1999

29. Spiegelmann R, Nissim O, Menhel J, et al: Linear accelerator radiosurgery for meningiomas in and around the cavernous sinus. Neurosurgery 51:1373-1380, 2002

30. Stafford SL, Pollock BE, Foote RL, et al: Meningioma radiosurgery: tumor control, outcomes, and complications among 190 consecutive patients. Neurosurgery 49:1029-1038, 2001

31. Subach BR, Lunsford LD, Kondziolka D, et al: Management of petroclival meningiomas by stereotactic radiosurgery. Neurosurgery 42:437-445, 1998

32. Taylor BW Jr, Marcus RB Jr, Friedman WA, et al: The meningioma controversy: postoperative radiation therapy. Int J Radiat Oncol Biol Phys 15:299-304, 1988

33. Tishler RB, Loeffler JS, Lunsford LD, et al: Tolerance of cranial nerves of the cavernous sinus to radiosurgery. Int J Radiat Oncol Biol Phys 27:215-221, 1993

34. Vernimmen FJ, Harris JK, Wilson JA, et al: Stereotactic proton beam therapy of skull base meningiomas. Int J Radiat Oncol Biol Phys 49:99-105, 2001

35. Villavicencio AT, Black PM, Shrieve DC, et al: Linac radiosurgery for skull base meningiomas. Acta Neurochir 143: $1141-1152,2001$

Manuscript received March 28, 2003.

Accepted in final form April 14, 2003.

Address reprint requests to: Antonio A. F. De Salles, M.D., Ph.D., 200 UCLA Medical Plaza, Room 504, Los Angeles, California 90095. email: adesalles@mednet.ucla.edu. 